\title{
Internet Use for Health-Care Information by Subjects With COPD
}

\author{
Cionéia K Delgado PT MSc, Mariana R Gazzotti PT PhD, Ilka L Santoro MD, \\ Andrea K Carvalho PT PhD, José R Jardim MD, and Oliver A Nascimento MD
}

\begin{abstract}
BACKGROUND: Although the internet is an important tool for entertainment, work, learning, shopping, and communication, it is also a possible source for information on health and disease. The aim of this study was to evaluate the proportion of subjects with COPD in São Paulo, Brazil, who use the internet to obtain information about their disease. METHODS: Subjects $(N=382)$ with COPD answered a 17-question survey, including information regarding computer use, internet access, and searching for sites on COPD. Our sample was distributed according to the socioeconomic levels of the Brazilian population (low, 17.8\%; medium, 66.5\%; and high, 15.7\%). RESULTS: Most of the subjects in the sample were male $(62.6 \%)$, with a mean age of $67.0 \pm 9.9 \mathrm{y}$. According to Global Initiative for Chronic Obstructive Lung Disease (GOLD) stages, $\mathbf{7 4 . 3 \%}$ of the subjects were in stage II or III. In addition, $51.6 \%$ of the subjects had a computer, $49.7 \%$ accessed the internet, and $13.9 \%$ used it to search for information about COPD. The internet was predominantly accessed by male (70.3\%) and younger (64.6 $\pm 9.5 \mathrm{y}$ of age) subjects compared with female $(29.7 \%, P=.04)$ and older $(67.5 \pm 9.6$ y of age, $P<.007)$ subjects. Searching for information about COPD on the internet was associated with having a computer (5.9-fold), Medical Research Council dyspnea level 1 (5.3-fold), and high social class (8.4-fold). The search for information on COPD was not influenced by GOLD staging. CONCLUSIONS: A low percentage of subjects with COPD in São Paulo use the internet as a tool to obtain information about their disease. This search is associated with having a computer, low dyspnea score, and high socioeconomic level. Key words: COPD; internet; health education. [Respir Care 2015;60(9):1276-1281. (C) 2015 Daedalus Enterprises]
\end{abstract}

\section{Introduction}

COPD is a progressive inflammatory disease characterized by chronic respiratory symptoms, exacerbation of symptoms, and activity limitation and is becoming one of the greatest health problems worldwide. ${ }^{1}$ This disease is expected to be the third leading cause of death in the world by 2020 and to move from twelfth to fifth place in the disability-adjusted life year. Adherence to treatment is es-

The authors are affiliated with the Respiratory Division, Pulmonary Rehabilitation Center, Escola Paulista de Medicina (EPM), Universidade Federal de São Paulo (UNIFESP), São Paulo, Brazil.

The authors have disclosed no conflicts of interest.

Correspondence: José R Jardim MD, Disciplina de Pneumologia, EPM, UNIFESP, Rua Botucatu, $7403^{\circ}$ andar, 04023-062 São Paulo, SP, Brazil. E-mail: jardimpneumo@gmail.com.

DOI: $10.4187 /$ respcare. 03716 sential for therapeutic success, ${ }^{2}$ and one of the important factors in patient adherence to treatment is disease knowledge. Therefore, patient education regarding the disease and its evolution and treatment is essential in the management of chronic diseases.

According to the National Cancer Institute, the internet has become a quick and easy tool for providing health-care information. ${ }^{3}$ Patient interest in obtaining information about disease via the internet varies from 8 to $60 \%$ depending on the problem: breast cancer $(43 \%),{ }^{4}$ chronic pain $(39 \%),{ }^{5}$ cardiovascular disease $(20 \%),{ }^{6}$ colorectal cancer $(8 \%),{ }^{7}$ burns $(12 \%),{ }^{8}$ infertility $(58 \%),{ }^{9}$ and lung cancer $(16 \%)^{10}$, as well as country and population.

This is not a new research phenomenon, and although the results should not be different for patients with COPD, they cannot be generalized. Although there are several sites with information on COPD, there has been no study to date that has assessed the use of the internet as a means of providing health-care information to patients. Thus, the purpose of this study was to estimate the proportion of 
subjects with COPD who have a computer and access the internet to find information about this disease in the city of São Paulo, Brazil.

\section{Methods}

This study was approved by the local research ethics Committee (1360/08), and all of the participants gave written consent. The inclusion criteria were a diagnosis of COPD according to the Global Initiative for Chronic Obstructive Lung Disease (GOLD) ${ }^{2}$ and clinical stability, including no exacerbations during the prior 4 weeks. Subjects with other unstable respiratory diseases or comorbidities were excluded.

Out-patients with COPD were interviewed and classified by socioeconomic level according to Brazilian economic classification criteria, including information regarding the education level of the head of the household and purchasing capacity (eg, number of home appliances, cars, and household help). The subjects were then grouped into 3 economic levels: high, medium, and low. To obtain information related to the participants' access to computers and the internet and their search for information, a survey including 17 questions was developed. Subjects with COPD were interviewed by an experienced interviewer (CKD) who was trained not to influence their responses.

Spirometry was performed according to European Respiratory Society ${ }^{11}$ and Brazilian Thoracic Society ${ }^{12}$ criteria for diagnostic confirmation and staging of COPD. The Medical Research Council dyspnea scale ${ }^{13}$ was used to assess the intensity of the subjects' dyspnea.

\section{Statistical Analysis}

Numerical data are presented as mean $\pm \mathrm{SD}$, and categorical data are presented as absolute and percent values. The Student $t$ test was used for comparisons between 2 means of numerical variables. The chi-square test or Fisher exact test was used to assess association between 2 categorical variables, such as internet access, economic class, disease severity, and respiratory symptoms. In the search for information about COPD, binary logistic regression was used to analyze the influence of independent variables.

As this was the first study to assess internet access in Brazil, we conducted a pilot study with 170 subjects to estimate sample size, and we found that $40 \%$ of subjects accessed the internet. Using the $40 \%$ rate and adopting a CI of $95 \%$, we determined that 369 subjects were necessary as a sample to perform this study. $P<.05$ was interpreted as indicative of statistically significant differences.

\section{QUICK LOOK}

\section{Current knowledge}

COPD is the world's fourth leading cause of mortality and is projected to be the fifth leading disease in morbidity impact by 2020 . The preponderance of patient management is done in primary care and at home. Education of patients regarding their disease, its evolution, and its treatment is essential in the management of patients with COPD.

\section{What this paper contributes to our knowledge}

In São Paulo, a low percentage of subjects with COPD use the internet to obtain information about their disease. This finding is similar to results of studies carried out in other countries and with other chronic diseases. Higher economic level, lower dyspnea intensity, and computer availability were found to be the independent predictors for access.

\section{Results}

Among the 400 people contacted, 382 consented to participate in the study: 239 (62.6\%) were male, and 143 $(37.4 \%)$ were female. The mean age was $67.0 \pm 9.9 \mathrm{y}$. Demographic data are provided in Table 1. The subjects were classified according to their economic status, with most of them belonging to the middle class (66.5\%). Most of the subjects were in GOLD stages II (40.9\%) and III (33.4\%).

Of the 382 subjects in the study, $51.6 \%$ reported having a computer at home, $49.7 \%$ accessed the internet, and $48 \%$ of those subjects searched online for information on COPD. Among the 185 subjects (48.4\%) who had no computer at home, $7 \%$ accessed the internet elsewhere, and $46 \%$ sought information about COPD elsewhere (Fig. 1). Thus, only 53 (13.9\%) of all COPD subjects evaluated in our study sought information about their disease on the internet.

The mean age of subjects who accessed the internet and searched for information on COPD was lower than that of subjects who did not, but the difference ( 3 y) had no clinical implication. Table 2 shows the characteristics of the subjects who had a computer, accessed the internet, and searched for information on COPD. Among those who had a computer, men accessed the internet more than women $(P<.05)$. No statistical differences were observed regarding the presence of respiratory symptoms, having a computer, access to the internet, and searching for information about COPD.

In the logistic regression model adjusted for variables that could influence the search for information on COPD 
Table 1. Characteristics of the Study Population

\begin{tabular}{lr}
\hline \multicolumn{1}{c}{ Variable } & \multicolumn{1}{c}{ Values } \\
\hline $\begin{array}{l}\text { Age, mean } \pm \text { SD y } \\
\text { Sex, } n(\%)\end{array}$ & $67.0 \pm 9.9$ \\
$\quad$ Female & $143(37.4)$ \\
$\quad$ Male & $239(62.6)$ \\
GOLD stage, $n(\%)$ & \\
I & $20(5.3)$ \\
II & $152(40.9)$ \\
III & $124(33.4)$ \\
IV & $76(20.4)$ \\
MRC dyspnea scale score, $n(\%)$ & \\
1 & $16(4.2)$ \\
2 & $98(25.7)$ \\
3 & $111(29.1)$ \\
4 & $60(15.7)$ \\
5 & $97(25.4)$ \\
Economic status, $n(\%)$ & $60(15.7)$ \\
High & $254(66.5)$ \\
Middle & $68(17.8)$ \\
Low & \\
& \\
$N=382: 372$ subjects underwent spirometry testing, and 10 subjects were not able to repeat \\
spirometry. \\
GOLD $=$ Global Initiative for Chronic Obstructive Lung Disease \\
MRC $=$ Medical Research Council \\
\hline
\end{tabular}

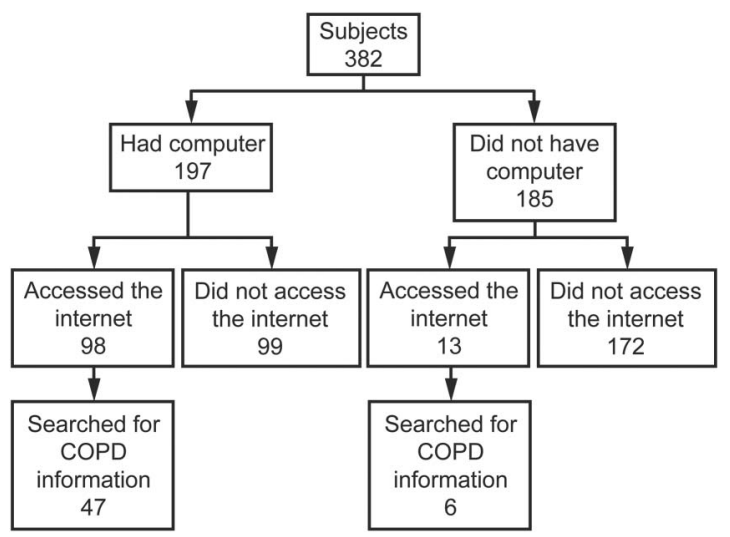

Fig. 1. Flow chart.

on the internet, we found that having a computer, the presence of low dyspnea intensity, and inclusion in the high economic class resulted in significant differences. With regard to searching for COPD information on the internet, the odds ratios for subjects with a computer, dyspnea grade 1, and inclusion in the high economic class were 5.9, 5.3, and 8.4 times higher, respectively (Table 3).

Subjects who searched for information on COPD reported accessing the internet from their own homes $(n=46$, $86.8 \%)$, homes of friends or family members $(n=4$, $7.5 \%)$, workplace $(n=2,3.8 \%)$, and internet cafes $(n=1$, $1.9 \%)$. In terms of weekly frequency, subjects accessed the internet 1-2 (26.4\%), 3-4 (13.2\%), 5-7 (13.2\%), or $>7$ (47.2\%) times. When asked about the importance of the internet to their disease knowledge, they answered that the internet helped them a lot (58.5\%), moderately (22.6\%), little $(7.5 \%)$, or not at all $(11.3 \%)$ in their learning.

Among subjects with no access to the internet, 157 (57.9\%) expressed their wish to obtain information about the disease through the internet. A large proportion of subjects who did not seek information about COPD on the internet answered that the information provided by the physician was sufficient (Fig. 2).

The web sites accessed by subjects when seeking information on COPD were www.dpoc.org.br (35.9\%), www. pacientes.com.br (25.6\%), www.abcdasaude.com.br (10.2\%), www.pulmonar.org.br (7.7\%), www.respireeviva.com.br (7.7\%), www.unifesp.br (5.1\%), and www.lesf.org (2.5\%). These sites were rated as good or excellent quality. According to the subjects, having no computer $(n=133,49.1 \%)$, not knowing how to use a computer or the internet $(n=71$, $26.2 \%)$, having no interest $(n=36,13.3 \%)$, and disliking computers or the internet $(n=31,11.4 \%)$ were the main barriers to accessing the internet.

\section{Discussion}

The internet has become one of the greatest means of communication. Every day, people access the internet for entertainment, work, communication, shopping, studying, and obtaining information on health and diseases. ${ }^{14-16}$ Efforts to increase patient information and to involve patients actively in making medical decisions are increasing worldwide. Educating patients with regard to COPD is crucial for better disease management, greater treatment adherence, better symptom control, and consequently, better quality of life. ${ }^{17}$ The aim of this study was to assess the proportion of subjects with COPD in São Paulo who access the internet for information about their disease. Only $13.9 \%$ of the 382 subjects with COPD who were interviewed used the internet to search for information about their disease, and this access was directly related to high economic level (including education level), low-grade dyspnea, and having a computer at home.

A study of lung cancer subjects in the United States showed they had low interest in seeking information about their disease on the internet. ${ }^{10}$ Only $16 \%$ of the subjects sought information about their disease on the internet, and their search was also related to higher income and higher education level. Another study conducted in the United States with subjects undergoing cardiac surgery showed that only $21 \%$ searched for medical information on the internet. ${ }^{18}$ Education level was the most important factor. A low education level and female sex seem to be the main limiting factors in accessing internet information ${ }^{19}$; 
Table 2. Association Between Subjects With a Computer With Internet Access and Search for Information on COPD

\begin{tabular}{|c|c|c|c|}
\hline \multirow[b]{2}{*}{ Parameter } & \multicolumn{3}{|c|}{ Subject Characteristics } \\
\hline & $\begin{array}{l}\text { Subjects With a Computer } \\
\qquad(n=197)\end{array}$ & $\begin{array}{l}\text { Subjects With Access } \\
\text { to the Internet }(n=111)\end{array}$ & $\begin{array}{l}\text { Subjects Who Searched for } \\
\text { COPD Information }(n=53)\end{array}$ \\
\hline \multicolumn{4}{|l|}{ Sex, $n(\%)$} \\
\hline Female & $65(45)$ & 33 (29.7) & $18(34)$ \\
\hline Male & $132(55)$ & $78(70.3)$ & $35(66)$ \\
\hline \multicolumn{4}{|l|}{ Economic status, $n(\%)$} \\
\hline High & $55(27.9)$ & $39(35.1)$ & $24(45.3)$ \\
\hline Middle & $130(66)$ & $66(59.5)$ & $27(50.9)$ \\
\hline Low & $12(6.1)$ & $6(5.4)$ & $2(3.8)$ \\
\hline \multicolumn{4}{|c|}{ MRC dyspnea scale score, $n(\%)$} \\
\hline 1 & $8(4.1)$ & $9(8.1)$ & $5(9.4)$ \\
\hline 2 & $46(23.4)$ & $28(25.2)$ & $11(20.8)$ \\
\hline 3 & $60(30.5)$ & $32(28.8)$ & $14(26.4)$ \\
\hline 4 & $31(17.5)$ & $17(15.3)$ & $12(22.6)$ \\
\hline 5 & $52(26.4)$ & $25(22.5)$ & $11(20.8)$ \\
\hline
\end{tabular}

Table 3. Binary Logistic Regression Analysis of Predictors of Information Search by Subjects With COPD

\begin{tabular}{|c|c|c|c|c|c|}
\hline Independent Variable & Coefficient B & SE & $P$ & $\operatorname{Exp}(B)$ & $95 \% \mathrm{CI}$ \\
\hline Have a computer & 1.778 & 0.487 & .001 & 5.919 & $2.9-15.4$ \\
\hline Sex & -0.077 & 0.356 & .83 & 0.92 & $0.5-1.9$ \\
\hline Age & 0.001 & 0.017 & .96 & 1.00 & $0.97-1.1$ \\
\hline MRC dyspnea scale score 1 & 1.671 & 0.707 & .02 & 5.31 & $1.3-21.2$ \\
\hline High economic status & 2.140 & 0.809 & .008 & 8.49 & $1.7-41.5$ \\
\hline
\end{tabular}

$\overline{\mathrm{MRC}}=$ Medical Research Council

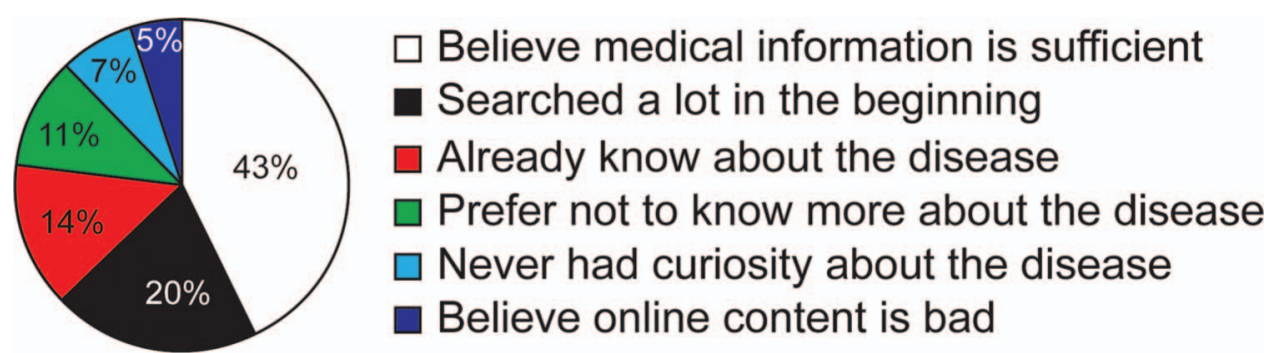

Fig. 2. Reasons given by subjects with COPD explaining why they did not search for COPD information online.

however, internet access seems to be related to disease severity. ${ }^{20}$

In our regression model, we found that subjects with lower degrees of dyspnea were more likely to seek information about COPD on the internet, possibly because patients in the initial phase of their disease usually have high expectations regarding their treatment. We found no association between age and searching for information. However, in another study, British colorectal cancer subjects younger than 60 y of age were more likely to use the internet to obtain medical information. ${ }^{7}$ Similarly, Swiss subjects 25-44 y of age were more likely to search for information on the internet ${ }^{20}$; however, the comparison is not equivalent, as this group was much younger than our subjects (67.9 y old) and the subjects in the British study. ${ }^{7}$

Ease of access to the internet can be a factor related to searching for information. In our study, only $13.9 \%$ of subjects sought information on COPD. However, this rate increased to $48 \%$ when the evaluation was restricted 


\section{Internet Health-Care Information for Subjects With COPD}

to subjects who had internet access. Previous studies have not discriminated internet access by population subgroups; therefore, it is difficult to make comparisons with our results. However, a Dutch study that assessed subjects with pain showed that $39 \%$ of them sought specific information about this condition on the internet. ${ }^{21}$ Similar to our study, the rate increased to $58 \%$ when subjects with internet access were evaluated as a subgroup. This high percentage of subjects seeking information about pain in The Netherlands might be related to the fact that $83 \%$ of the general population has internet access.

Not having a computer or internet access (49.1\%), not knowing how to use a computer $(26.2 \%)$, having no interest $(13.3 \%)$, and disliking computers or the internet $(11.4 \%)$ were the main reasons that our subjects provided for not using the internet. Our results are similar to those of a United States study in which 53\% of cancer subjects claimed lack of a computer as their main barrier to seeking information on the internet. ${ }^{10}$ Moreover, in a study of subjects who underwent thoracic surgery in a rural area of the United States, the high cost of internet services was a barrier to internet access. ${ }^{22}$

Regarding quality of information found on the internet, our subjects rated the sites they accessed as being of good or excellent quality. In a Dutch study, $43 \%$ of the subjects interviewed also rated the quality of the information as good. ${ }^{5}$

In this study, $43 \%$ of the subjects said that they did not access the internet because they considered the information provided by their physicians to be sufficient, and 5\% considered the internet content to be incorrect. In a study conducted in England, $80 \%$ of the subjects preferred to receive their medical information directly from their physicians; only $19 \%$ searched for information on the internet. ${ }^{21}$ Similarly, in a study conducted in The Netherlands, the subjects generally preferred to have their medical information provided by their physicians, despite having access to the internet. ${ }^{5}$ Subjects in a study conducted in Taiwan stated that they did not seek medical information on the internet due to lack of time, lack of skill in accessing it, motivation, or even dissatisfaction with the information, considering it to be unreliable. ${ }^{23}$

In our study, however, a proportion of the subjects $(57.9 \%)$ without access to the internet expressed a wish to obtain information about their disease via the internet. This group believed that they could have better control over their symptoms if they knew more about the disease. They also stated that if the physician were to recommend a site, a family member could access the information and pass it along to the subjects. This finding is similar to that described in the literature, in which $60-78 \%$ of subjects wished to have an internet source of information about the disease. ${ }^{10,24,25}$ These data indicate that the internet will become even more important in the coming years. Although barriers to internet access still exist, we believe that they will decrease with time as technology progresses, making health-care information on the internet more common.

We were unable to quantify the impact of internet use on the doctor-patient relationship or on self-management of the disease. We also did not measure the amount of disease knowledge acquired via the internet due to the difficulty in isolating other media, which generated confounding factors for the analyses.

\section{Conclusions}

Our study shows that in São Paulo, a low percentage of subjects with COPD use the internet to obtain information about their disease. Although this finding is similar to results of studies carried out in other countries and with other diseases, it is the first time that this information has been provided for COPD. Higher economic level, lower dyspnea intensity, and computer availability were found to be the independent predictors for access. Although the percentage of subjects who searched for information online was still low, nearly $60 \%$ of the subjects were interested in acquiring knowledge about their disease via the internet. This finding indicates that barriers to internet access tend to decrease as patient interest increases and that use of this medium will increase. Therefore, healthcare professionals should encourage their patients to search for information on the internet, refer them to reliable sites, and be available to clarify doubts that might arise. It is still the responsibility of the health-care professional to determine patients' needs, the best method to present this education, and the best method to evaluate the effects. internet cost is likely to remain a barrier unless free access becomes available. Finally, we believe that this information might be generalizable for patients with COPD from other countries, as the profile of our subjects was very similar to those described in the literature, and they were studied according to socioeconomic level.

\section{REFERENCES}

1. Jardim JR, Nascimento OA, Oliveira JC. II Consenso Brasileiro sobre doença pulmonar obstrutiva crônica (DPOC). J Bras Pneumol 2004;30:S1-S42. Article in Portuguese.

2. Global Initiative for Chronic Obstructive Lung Disease. Global strategy for the diagnosis, management, and prevention of chronic obstructive pulmonary disease. Updated 2013. http://www.goldcopd.org. Accessed May 19, 2015.

3. National Institute of Cancer, National Institutes of Health. Citado em: 04 de janeiro de 2012. http://www.cancer.gov. Accessed May 19, 2015.

4. Pereira JL, Koski S, Hanson J, Bruera ED, Mackey JR. Internet usage among women with breast cancer: an exploratory study. Clin Breast Cancer 2000;1(2):148-153; discussion 154-155. 


\section{InTERnEt Health-CARe Information For SubJects With COPD}

5. de Boer MJ, Versteegen GJ, van Wijhe M. Patients use of the Internet for pain-related medical information. Patient Educ Couns 2007; 68(1):86-97.

6. Neubeck L, Ascanio R, Bauman A, Briffa T, Clark AM, Freedman B, Redfern J. Planning locally relevant Internet programs for secondary prevention of cardiovascular disease. Eur J Cardiovasc Nurs 2011;10(4):213-220.

7. Powell SM, McStay RA, Hanson JM, Plusa SM. Use of the Internet by colorectal cancer patients. Colorectal Dis. 2006;8(1):62-63.

8. Rea S, Lim J, Falder S, Wood F. Use of the Internet by burns patients, their families and friends. Burns 2008;34(3):345-349.

9. Rawal N, Haddad N. Use of Internet in infertility patients. Internet J Gynecol Obstet 2005;5(2).

10. Peterson MW, Fretz PC. Patient use of the Internet for information in a lung cancer clinic. Chest 2003;123(2):452-457.

11. Miller MR, Hankinson J, Brusasco V, Burgos F, Casaburi R, Coates A, et al. Standardisation of spirometry. Eur Respir J 2005;26(2):319-338.

12. Sociedade Brasileira de Pneumologia e Tisiologia. Diretrizes para testes de função pulmonar. J Bras Pneumol 2002;28(3):1-221. Article in Portuguese.

13. Kovelis D, Segretti NO, Probst VS, Lareau SC, Brunetto AF, Pitta F. Validation of the Modified Pulmonary Functional Status and Dyspnea Questionnaire and the Medical Research Council scale for use in Brazilian patients with chronic obstructive pulmonary disease. J Bras Pneumol 2008;34(12):1008-1018.

14. Harrison JP, Lee A. The role of e-health in the changing healthcare environment. Nurs Econ 2006;24(6):283-288.

15. Coye MJ, Haselkorn A, DeMello S. Remote patient management: Technology-enabled innovation and evolving business models for chronic disease care. Health Affairs 2009;28(1):126-135.
16. Black AD, Car J, Pagliari C, Anandan C, Cresswell K, Bokun T, et al. The impact of eHealth on the quality and safety of healthcare: a systematic overview. PLoS Med 2011;8(1):e1000387.

17. Duplaga M. The acceptance of e-health solutions among patients with chronic respiratory conditions. Telemed J E Health 2013;19(9): 683-691.

18. Murero M, D'Ancona G, Karamanoukian H. Use of the Internet by patients before and after cardiac surgery: an interdisciplinary telephone survey. J Med Internet Res 2001;3(3):e27.

19. Helft PR, Eckles RE, Johnson-Calley CS, Daugherty CK. Use of the Internet to obtain cancer information among cancer patients at an urban county hospital. J Clin Oncol 2005;23(22):4954-4962.

20. Jeannot JG, Froehlich F, Wietlisbach V, Burnand B, Terraz O, Vader JP. Patient use of the Internet for health care information in Switzerland. Swiss Med Wkly 2004;134(21):307-312.

21. Dolan G, Iredale R, Williams R, Amee J. Consumer use of the Internet for health information: a survey of primary care patients. Int J Consum Stud 2004;28(2):147-153.

22. Quin J, Stams V, Phelps B, Boley T, Hazelrigg S. Interest in Internet lung cancer support among rural cardiothoracic patients. J Surg Res 2010;160(1):35-39.

23. Hsu LL. An exploratory study of Taiwanese consumers' experiences of using health-related websites. J Nurs Res 2005;13(2): 129-140.

24. Ross J, Chapman C, Murray C. How much interest is the Internet to patients? Sex Transm Infect 2000;76(5):393-394.

25. O'Connor JB, Johanson JF. Use of the Web for medical information by a gastroenterology clinic population. JAMA 2000;284(15):19621964. 